\title{
EPIDEMIOLOGÍA ENFERMERA DEL DUELO EN CANARIAS
}

\author{
Martín Rodríguez-Álvaro* \\ Universidad de La Laguna
}

\section{RESUMEN}

El duelo es un proceso natural y autolimitado de adaptación a una nueva realidad que surge tras una pérdida significativa, real o percibida, con un amplio abanico de manifestaciones que producen un impacto en la salud del doliente. Objetivo: conocer e identificar, desglosado por gradiente diagnóstico, la prevalencia del duelo en Canarias, así como las necesidades de cuidado previas al diagnóstico de duelo de los dolientes. Resultados: se identifican significativamente más mujeres que hombres en las tres etiquetas diagnósticas a estudio. Los pacientes con complicaciones de duelo presentan previamente depresión, ansiedad y riesgo de soledad. El resultado de la valoración del patrón es paradójico. Conclusión: el duelo es un fenómeno multidimensional que difícilmente puede ser valorado desde una herramienta reduccionista como los patrones funcionales. Se debe profundizar en la alta prevalencia de complicaciones en el duelo de la mujer, asociándolo a sus cargas de cuidado y vulnerabilidad previa. La asociación entre la soledad y el duelo complicado es evidente y el papel de las enfermeras comunitarias en estos pacientes puede ser clave. Es necesario seguir investigando acerca de las necesidades de cuidados en los dolientes, con y sin complicaciones, así como en sus factores predictores y protectores.

Palabras Clave: duelo, proceso de enfermería, atención primaria.

\section{NURSING EPIDEMIOLOGY OF GRIEF IN THE CANARY ISLANDS}

\section{Abstract}

Grief is a natural and self-limited process of adaptation to a new reality that arises after a significant loss, real or perceived, with a wide range of manifestations that produce an impact on the health of the mourner. Objective: To know and identify, broken down by diagnostic gradient, the prevalence of mourning in the Canary Islands, as well as the needs of care before the diagnosis of mourning of the mourners. Results: Significantly, more women than men are identified in the three diagnostic labels under study. Patients with bereavement complications previously presented depression, anxiety, and risk of loneliness. The result of the pattern assessment is paradoxical. Conclusion: Grief is a multidimensional phenomenon that can hardly be assessed from a reductionist tool such as functional patterns. The high prevalence of complications in women's grief should be studied in-depth, associating it with their previous burdens of care and vulnerability. The association between loneliness and complicated grief is evident, and the role of community nurses in these patients may be essential. Further research is needed on the care needs of bereaved persons, both complicated and uncomplicated, as well as on their predictive and protective factors.

KeYwords: Grief, Nursing process, Primary care. 


\section{INTRODUCCIÓN}

En la literatura especializada el proceso de duelo ha sido definido por muchos autores desde ópticas muy diferentes. Buscando una definición que interprete el proceso como un fenómeno complejo y heterogéneo, el duelo puede ser definido como «un proceso natural y autolimitado de adaptación a una nueva realidad que surge tras una pérdida significativa, real o percibida, con un amplio abanico de manifestaciones que producen un impacto en la salud del doliente». La taxonomía diagnóstica NANDA-I, la más extendida en la práctica enfermera, proporciona una terminología estandarizada de diagnósticos de enfermería, una clasificación sistemática de los fenómenos que definen el conocimiento enfermero ${ }^{1}$. Los diagnósticos enfermeros relacionados con el duelo han sido modificados a lo largo de las últimas décadas a la par que ha ido cambiado y evolucionando la concepción del duelo en general. Actualmente distingue entre tres etiquetas diagnósticas: Duelo, Riesgo de Duelo Complicado y Duelo Complicado. Estas etiquetas se encuadran en el dominio 9, Afrontamiento/tolerancia al estrés, referido a la convivencia con eventos y procesos vitales, perteneciendo, a su vez, a la clase 2, Respuestas de afrontamiento, descritas como procesos necesarios para la gestión del estrés del entorno. Estos diagnósticos aportan un nivel de evidencia de 2.1, esto es, aceptado para su publicación con etiqueta, definición, características definitorias o factores de riesgo, factores relacionados y bibliografía (se describen tres niveles de evidencia: Recibido para su desarrollo; Aceptado para su publicación e inclusión en la Taxonomía; Soporte clínico).

La valoración enfermera consiste en recabar, analizar y valorar la información sobre el estado de salud y busca evidencias de funcionamiento anormal o factores de riesgo que pueden generar problemas de salud ${ }^{2}$. Gordon asocia las etiquetas relacionadas con el duelo (anticipado y funcional) con el Patrón Funcional de Salud 8, rol Relaciones, indicando que este patrón «describe el patrón de compromisos del rol y relaciones. Incluye la percepción del individuo de los principales roles y responsabilidades en su situación vital actual. También comprende la satisfacción o problemas con la familia, el trabajo, las relaciones sociales y las responsabilidades relacionadas con estos roles» ${ }^{3}$.

* Doctor, enfermero de Atención Primaria, Área de Salud de La Palma. Servicio Canario de Salud. Profesor asociado del Departamento de Enfermería de la Universidad de La Laguna. Miembro del Grupo de Investigación Cuidados al Final de la Vida (CUFINVIDA). Universidad de La Laguna. Editor de la Revista Ene de Enfermería.

${ }^{1}$ Herdman, T.H. y Kamitsuru, S. (eds.). NANDA International Nursing Diagnoses: Definitions \& Classification, 2018-2020. Oxford: Wiley Blackwell. 2017.

2 Alfaro-LeFevre, R. Aplicación del proceso enfermero fomentar el cuidado en colaboración. Elsevier España; 2003. 308 p.

${ }^{3}$ Gordon, M. Manual of Nursing Diagnosis, 1988-89: Including All Diagnostic Categories Approved by the North American Nursing Diagnosis Association. C.V. Mosby; 1989. 378 p. 
Los diagnósticos de duelo han sido modificados a lo largo de las últimas décadas a la par que ha ido cambiado y evolucionando la concepción del duelo en general. Actualmente NANDA-I distingue entre tres etiquetas diagnósticas: Duelo, Riesgo de Duelo Complicado y Duelo Complicado. Estas etiquetas se encuadran en el dominio 9, Afrontamiento/tolerancia al estrés, referido a la convivencia con eventos y procesos vitales, perteneciendo, a su vez, a la clase 2, Respuestas de afrontamiento, descritas como procesos necesarios para la gestión del estrés del entorno. Estos diagnósticos aportan un nivel de evidencia de 2.1, esto es, aceptado para su publicación con etiqueta, definición, características definitorias o factores de riesgo, factores relacionados y bibliografía (se describen tres niveles de evidencia: Recibido para su desarrollo; Aceptado para su publicación e inclusión en la Taxonomía; Soporte clínico).

La etiqueta diagnóstica Duelo, antes Duelo Anticipado, se incluye en 1980 en la taxonomía diagnóstica NANDA-I. Este diagnóstico se revisa en las ediciones de 1996, 2006 y 2017, donde es definido como el «Proceso complejo normal que incluye respuestas y conductas emocionales, físicas, espirituales, sociales e intelectuales mediante las que las personas, familias y comunidades incorporan en su vida diaria una pérdida real, anticipada o percibida». Usando como referencia los trabajos de Ott ${ }^{4}$ y Hogan, Worden y Schmidt se da un paso hacia el entendimiento del proceso de duelo desde una óptica menos conservadora. Las revisiones de este diagnóstico enfermero añaden características definitorias que tienden a normalizar el proceso ${ }^{6}$.

Riesgo de Duelo Complicado se incluye en 2004 en la taxonomía. Este diagnóstico, aprobado en 2004, es revisado en la edición de 2006, 2013 y 2017, donde se define como «Susceptible de sufrir un trastorno que ocurre tras la muerte de una persona significativa, en el que la experiencia del sufrimiento que acompaña al luto no sigue las expectativas normales y se manifiesta en un deterioro funcional».

Finalmente, Duelo Complicado, antes Duelo Disfuncional, se incluye en 1980 en la taxonomía diagnóstica. Este diagnóstico se revisa en las ediciones de 1986, 2004, 2006 y 2017, donde se define como el «Trastorno que ocurre tras la muerte de una persona significativa, en el que la experiencia del sufrimiento que acompaña al luto no sigue las expectativas normales y se manifiesta en un deterioro funcional». Es de destacar que esta etiqueta diagnóstica enfermera no utiliza una escala temporal para su detección, pese a que, como refiere Carpenito, «las reacciones agudas a una pérdida no deben denominarse disfuncionales, sea cual sea su gravedad. El

${ }^{4}$ Отт, СH. The impact of complicated grief on mental and physical health at various points in the bereavement process. Death Stud, abril de 2003;27(3): 249-72.

5 An Empirical Study of the Proposed Complicated Grief Disorder Criteria, Nancy S. Hogan, J. William Worden, Lee A. Schmidt, 2004 [Internet]. [Citado 14 de febrero de 2020]. Disponible en https://journals.sagepub.com/doi/abs/10.2190/GX7H-H05N-A4DN-RLU9?journalCode=omea.

${ }^{6}$ Rodríguez Alvaro, M., García Hernández, A.M. y Toledo Rosell, C. Hacia una visión constructivista del Duelo. Index de Enfermería [Internet]. septiembre de 2008 [citado 14 de febrero de 2020];17(3):193-6. Disponible en http://scielo.isciii.es/scielo.php?script=sci_abstract\&pi$\mathrm{d}=$ S1132-12962008000300009\&lng=es\&nrm=iso\&tlng=es. 
duelo disfuncional se caracteriza por una reacción perjudicial mantenida o prolongada. No se puede confirmar que es un duelo difuncional hasta varios meses o un año después de la pérdida ${ }^{7}$.

Los diagnósticos enfermeros pueden funcionar como descriptores clave de necesidades de cuidados poblacionales para el perfil de paciente crónico, sobre todo en aquellos con mayor nivel de complejidad. Un ejercicio sistemático de epidemiología enfermera permite crear redes centinela de necesidades de cuidados ${ }^{8}$. El propósito principal de este estudio es conocer e identificar, desglosado por gradiente diagnóstico, la prevalencia del duelo en Canarias, así como las necesidades de cuidado previas al diagnóstico de duelo de los dolientes. Para esto, se toman como elemento central las etiquetas diagnósticas enfermeras Duelo, Riesgo de Duelo Complicado y Duelo Complicado.

Esta investigación forma parte de un proyecto de investigación mayor, la tesis doctoral Epidemiología enfermera del duelo en Canarias ${ }^{9}$, del que ya se han publicado y difundido resultados. En concreto respecto a la prevalencia de las etiquetas diagnósticas a estudio y sus características diagnósticas ${ }^{10}$, abordando el plan de cuidados específico planificado por las enfermeras comunitarias en estos pacientes ${ }^{11}$, identificando las etiquetas diagnósticas predictoras de complicaciones en el proceso de duelo ${ }^{12}$, estudiando el impacto del duelo a través del lenguaje del cuidado $^{13}$ y reflexionando acerca de la necesidad de cambiar la visión enfermera de la pérdida y el duelo ${ }^{14}$.

2017.

7 Carpenito L.J. Diagnóstico de Enfermería. 15. a edición. Interamericana-McGraw-Hill.

${ }^{8}$ Brito P.R., Aguirre A., Duarte G. y Sánchez, M.B. Investigación epidemiológica en diagnósticos enfermeros. En Echevarría, P. Investigación en metodología y lenguajesen-fermeros. Barcelona: Elsevier, 187-199.

9 Rodríguez, M. Epidemiología enfermera del duelo en canarias. Universidad de La Laguna; 2017 [citado 9 de febrero de 2020]. Disponible en https:/www.educacion.es/ teseo/mostrarRef.do?ref $=1454574$.

${ }^{10}$ Rodríguez-Állvaro, M., Brito-Brito, P.R., García-Hernández, A.M., Aguirre-Jaime, A., Fernández-Gutiérrez, D.A. The Grieving Nursing Diagnoses in the Primary Healthcare Set- ting: The Grieving Nursing Diagnoses in the Primary Healthcare Setting. Int J Nurs Terminol Knowledge, enero de 2019;30(1): 34-42.

${ }^{11}$ Rodríguez-Álvaro, M., García-Hernández, A.M., Brito-Brito, P.R., Aguirre-Jaime, A., Fernández-Gutiérrez, D.Á. Intervenciones y criterios de resultado planificados por las enfermeras comunitarias en la atención al duelo en Canarias. Enfermería Clínica. julio de 2018;28(4): 240-246.

12 Rodríguez-Álvaro, M., Brito Brito, P.R., García Hernández, A.M. y FernánDEZ Gutiérrez, D.Á. «Etiquetas diagnósticas predictoras de duelo complicado». En XII Simposium Internacional AENTDE. Cádiz; 2018.

13 Rodríguez-Álvaro, M. «Impacto del duelo complicado. una lectura a través del lenguaje del cuidado». Revista Ene de Enfermería [Internet]. 4 de septiembre de 2019 [citado 9 de febrero de 2020]; 13(3). Disponible en http://www.e-ne-enfermeria.org/ojs/index.php/ENE/article/view/915.

${ }^{14}$ Rodríguez-Álvaro, M., García-Hernández, A.M. y Brito-Brito, P.R. Hacia un modelo enfermero para entender la pérdida y el duelo. Enfermería Clínica. noviembre de 2017;27(6): 397-398. Rodríguez-Álvaro, M., García-Hernández, A.M. y Brito-Brito, P.R. Grieving is 


\section{MATERIAL Y MÉTODO}

El diseño corresponde al de un estudio retrospectivo elaborado con los registros de la historia informatizada de Atención Primaria del Servicio Canario de la Salud, en pacientes con registro de las etiquetas diagnósticas enfermeras de Duelo, Riesgo de Duelo Complicado y Duelo Complicado en el periodo 2009-2014.

En Canarias, la atención comunitaria se organiza en 7 áreas de salud, que reúnen en total 105 zonas básicas de salud en las que trabajan alrededor de 1169 enfermeras. La población canaria en 2014 era de 2,1 millones de habitantes, estimándose que un $44 \%$ de los pacientes acude al menos una vez al año a consultas de enfermería. El módulo de metodología enfermera de la historia de salud electrónica de Atención Primaria en Canarias, Drago-AP, sigue la lógica del proceso enfermero. Utiliza como marco de valoración los patrones funcionales de salud de Marjory Gordon, empleando los lenguajes estandarizados NANDA-I, NOC y NIC para la planificación de cuidados.

Para la elaboración del estudio, se solicitaron oficialmente los registros de las historias clínicas de los dolientes al centro de Servicio de Tecnologías de la Información del Servicio Canario de Salud. La explotación de datos facilitada a los autores respeta el principio de confidencialidad y la protección de datos de carácter personal.

\section{ANÁLISIS ESTADÍSTICO}

Las características de la muestra se describen resumiendo las variables nominales con las frecuencias de sus categorías, las ordinales y de escala no normal con mediana y percentiles (P5-P95) y las de escala con distribución normal con media y desviación estándar (DT). Las prevalencias e incidencias se estiman en intervalos de confianza al 95\%. Las comparaciones entre los grupos conformados se realizan con la prueba Chi-cuadrado de Pearson para las variables nominales. Todas las pruebas se ejecutaron con ayuda del paquete estadístico IBM-SPSS $24.0^{\circ}$ para entorno operativo Windows XP Professional@.

\section{RESULTADOS}

Se identifican 9063 resultados con las etiquetas diagnósticas enfermeras a estudio: duelo, 6091 (67,71\%); duelo complicado, 2429 (26,8\%) o riesgo de duelo complicado, 543 (6,0\%). Entre los años 2010 y 2014 el diagnóstico de Duelo se registra en un $78 \%$ de pacientes, independientemente del sexo y la edad. En el periodo anterior, 2005-2010, el diagnóstico más frecuentemente registrado es Duelo Compli-

not a Nursing Diagnosis. Int J Nurs Knowl. 13 de febrero de 2020; [Epub ahead of print] Disponible en https://doi.org/10.1111/2047-3095.12278. 
cado. El 80\% de las personas diagnosticadas son mujeres, con una edad de 71 (3987 años, de los que el $86 \%$ tenía 50 o más años y un $22 \%$, 80 años o más. Según los grupos de edad, el de mayor porcentaje fue el de personas con 65 o más años (63\%), seguido del de 35-64 (34\%) y el de 18-34 (3\%). La mediana de la edad en que se produjo el diagnóstico es de 70,24 años, mientras que la media fue de 67,32. Se hallan más asignaciones de Duelo Complicado en las mujeres $(\mathrm{p}<0,001)$, y en personas de 65 o más años $(\mathrm{p}<0,001)$. Igualmente se encuentran diferencias significativas en la asignación del Riesgo de Duelo Complicado, mayor en mujeres, y en el grupo de 18-34 ańos $(\mathrm{p}<0,001)$.

\section{ANTECEDEnTES MÉdicos}

El 4,6\% ( $n=101)$ de los pacientes diagnosticados de Duelo Complicado por la enfermera comunitaria son a su vez diagnosticados de Duelo Patológico por sus médicos de familia durante el año anterior o posterior al diagnóstico enfermero. Así mismo, en el $17 \%(\mathrm{n}=1.138)$ de los pacientes en los que la enfermera diagnostica Duelo o Riesgo de Duelo Complicado, el médico de familia diagnostica Duelo durante el ańo anterior o posterior al diagnóstico enfermero. El 2,8\% $(\mathrm{n}=67)$ de los Duelos Complicados son diagnosticados más de un año antes de Duelo Patológico por medicina de familia, mientras que en el 4,7\% ( $n=114)$ de los mismos son registrados como Duelo por medicina de familia en el mismo periodo. Se observan diferencias significativas $(\mathrm{p}<0,001)$ en aquellos pacientes en los que se identifican previamente un problema depresivo, como depresión mayor; trastorno adaptativo; depresión reactiva; depresión o síndrome depresivo; asociándose este con el desarrollo de Duelo Complicado. El 20\% $(\mathrm{n}=486)$ de los pacientes con Duelo Complicado presentan previamente este tipo de diagnósticos, frente al 17,2\% $(\mathrm{n}=1560)$ de los dolientes normales o en riesgo. Se observan así mismo diferencias entre aquellos pacientes diagnosticados previamente de Ansiedad ( $\mathrm{n}=1101 ; 12,10 \%)$. La sufren antes del diagnóstico de Riesgo de Duelo Complicado el 16,70\% ( $\mathrm{n}=91)$ de los pacientes, por un $12,40 \%(n=752)$ de Duelo y un $10,6 \%$ de Duelo Complicado $(n=178)$ $(\mathrm{p}=0,001)$. Se identifican casos aislados entre los dolientes de abuso de drogas y de fármacos $(n=17)$, sin presentar diferencias significativas entre Duelo y Duelo Complicado. En la presencia de insomnio previo al diagnóstico $(n=760 ; 8,45)$, no se evidencia significación estadística respecto a padecer Duelo Complicado. El $8 \%(\mathrm{n}=725)$ de los dolientes es derivado con anterioridad a Salud Mental, el 2,4\% $(\mathrm{n}=220)$ durante el año anterior y el 5,6\% ( $\mathrm{n}=550)$ más de un año antes. Encontramos diferencias según el gradiente diagnóstico de Duelo $(\mathrm{p}<0,001)$, son derivados previamente a este servicio el 10,65\% ( $\mathrm{n}=256)$ de los Duelos Complicados (fundamentalmente más de un año antes del diagnóstico $(7,3 \% ; n=177)$; mientras que en el resto de grupos es de un $7 \%(\mathrm{n}=469)$. El mismo día del diagnóstico se derivan a la Unidad de Salud Mental el 0,4\% ( $\mathrm{n}=32)$ de los dolientes. 
Durante el año previo al diagnóstico, en los pacientes diagnosticados de Duelo o Riesgo de Duelo Complicado, se valora más el PFS8, 13,1\% n=871, frente al 7,12\% $\mathrm{n}=173$, del grupo Duelo Complicado. Se aprecian diferencias en la asociación del resultado de la valoración con la asignación posterior de Duelo Complicado $(\mathrm{p}<0,001)$. En ese periodo, el 4,28\% ( $\mathrm{n}=104)$ de los pacientes diagnosticados de Duelo Complicado tienen como resultado de la valoración Alterado/Normal, 0,99\% ( $\mathrm{n}=24)$ Riesgo de Alteración y $1,85 \%(\mathrm{n}=45)$ Normal. En los dolientes no complicados, el resultado de la valoración es: 6,53\% $(n=433)$ Alterado/Normal, 2,62\% $(n=174)$ Riesgo de Alteración y 3,95\% ( $\mathrm{n}=262)$ Normal. Esta tendencia se mantiene en el periodo más de un año anterior al diagnóstico. En 2679 pacientes (29,66\%) se valora en el momento del diagnóstico el PFS8 teniendo como resultado Normal el 9,27\% ( $\mathrm{n}=837)$; Riesgo de alteración 5,56\% $(\mathrm{n}=502)$ y Alterado/Anormal 14,84\% $(\mathrm{n}=1340)$. Se utiliza más la valoración de este patrón entre los diagnosticados de Duelo normal o Riesgo de Duelo Complicado $(31,26 \% ; n=2066)$ que en los que es diagnosticados de Duelo Complicado $(25,30 \% ; \mathrm{n}=613)$. Entre los valorados y diagnosticados de Riesgo de Duelo Complicado o Duelo Complicado el mismo día, el resultado de la valoración de este patrón funcional se distribuye de la siguiente forma: Normal ( $\mathrm{n}=661 ; 31,99 \%)$; Riesgo de alteración ( $\mathrm{n}=413 ; 19,99 \%)$; Alterado/Anormal ( $\mathrm{n}=992 ; 48,02 \%)$; mientras, entre los que es diagnosticados de Duelo Complicado la distribución es la siguiente: Normal ( $\mathrm{n}=176 ; 28,71 \%)$; Riesgo de alteración $(\mathrm{n}=89 ; 14,52 \%)$; Alterado/Anormal $(\mathrm{n}=348 ; 56,77 \%)$. Se identifican un total de 193 etiquetas diagnósticas enfermeras. 125 de ellas se registraron en menos del $1 \%$ de los pacientes.

Los diagnósticos de enfermería más comunes registrados como antecedentes son: Conductas Generadoras de Salud ( $\mathrm{n}=2399 ; 26,51 \%)$; Dolor Agudo ( $\mathrm{n}=1975$; 21,80\%); Deterioro de la integridad cutánea $(\mathrm{n}=1743 ; 19,20 \%)$; Desequilibrio Nutricional: Ingesta superior a las necesidades $(\mathrm{n}=1669$; 18,405); Riesgo de Infección $(\mathrm{n}=1377 ; 15,20 \%)$; Disposición para mejorar el estado de inmunización ( $\mathrm{n}=1223$; 13,50\%); 00162_Disposición para mejorar la gestión de la propia salud $(\mathrm{n}=1177$; 135); Dolor Crónico ( $\mathrm{n}=1166 ; 12,90 \%)$; Riesgo de Caídas ( $\mathrm{n}=1153$; 12,70\%); Ansiedad ( $n=1080 ; 11,905)$; Insomnio ( $n=1057 ; 11,70 \%)$. Se identifican diferencias en las etiquetas diagnósticas según el sexo de los pacientes, registrándose más en mujeres: Desequilibrio Nutricional: ingesta superior a las necesidades $(\mathrm{p}=0,001)$; Incontinencia Urinaria de esfuerzo $(\mathrm{p}<0,001)$; Incontinencia urinaria de urgencia $(\mathrm{p}=0,003)$; Cansancio del rol del cuidador $(\mathrm{p}<0,001)$; Riesgo de Cansancio del rol de cuidador $(\mathrm{p}=0,006)$; Insomnio $(\mathrm{p}<0,001)$; Deprivación del sueño $(\mathrm{p}=0,043)$; Dolor agudo $(\mathrm{p}<0,001)$; Dolor crónico $(\mathrm{p}<0,001)$; Aflicción crónica $(\mathrm{p}<0,001)$. En hombres, se identifican más las etiquetas diagnósticas Riesgo de lesión ( $\mathrm{p}=0,003)$; Deterioro de la Integridad cutánea $(\mathrm{p}<0,001)$; Deterioro de la interacción social $(\mathrm{p}=0,039)$; Mantenimiento ineficaz de salud ( $\mathrm{p}=0,034)$; Déficit del autocuidado: vestido $(\mathrm{p}=0,005)$; Impotencia $(\mathrm{p}=0,024)$; Trastorno de los procesos de pensamiento $(\mathrm{p}=0,019)$; Disposición para mejorar el autocuidado ( $\mathrm{p}=0,042$ ); Disposición para mejorar el confort $(\mathrm{p}=0,005)$. Sólo en tres etiquetas (Riesgo de Traumatismo, Riesgo de Soledad y Duelo) se hallan diferencias significativas a favor de Duelo Complicado. 


\section{DISCUSIÓN}

La prevalencia de Duelo Complicado en Canarias identificada a través de los datos de este estudio ya ha sido analizada y discutida anteriormente. Para establecer comparaciones con investigaciones previas es necesario tener en cuenta que no hay consenso en la identificación, diagnóstico y definición de duelo complicado o prolongado. Se estima que alrededor del $6 \%$ de los dolientes se sitúa en alto riesgo de padecer complicaciones, $35 \%$ en riesgo moderado y el $60 \%$ estarían en bajo riesgo ${ }^{15}$. La literatura especializada muestra que finalmente entre el $2,5-20 \%$ de deudos sufren complicaciones en el proceso. Esta diferencia se relaciona con aspectos como la relación con la pérdida, edad y género, tipo de muerte, capacidad de darle significado, vulnerabilidad previa del doliente, pérdidas anteriores no resueltas, contexto sociocultural y criterios diagnósticos que se empleen.

De la alta identificación de duelo, duelo complicado y riesgo de duelo complicado en mujeres se ha hablado extensamente, siendo un resultado que se presenta habitualmente en la bibliografía consultada. Al estilo afrontamiento del duelo tradicionalmente identificado con las mujeres, sobre el que se construyen las teorías tradicionales del duelo, hay que sumar que el cuidado se sigue escribiendo en femenino. Más de la mitad de las personas con dependencia están atendidas por un cuidador familiar, siendo el $79 \%$ mujeres ${ }^{16}$. Uno de los factores predictores de complicaciones en el proceso de duelo es la dependencia del cuidador familiar primario respecto al paciente ${ }^{17}$. Las familias asumen en la mayor parte de los casos la totalidad de los cuidados de la persona dependiente, con todas las consecuencias que ello conlleva $^{18}$, y la mujer suele ser la que presta estos cuidados informales (se estima que el sistema sanitario formal dispensa sólo el $12 \%$ del tiempo total dedicado al cuidado $\left.^{19}\right)$. Paradójicamente, las mujeres suelen prestar atención a los miembros de la familia moribundos, pero cuando ellas mismas están muriendo, o padecen necesi-

15 Aoun, S.M., Breen, L.J., Howting, D.A., Rumbold, B., McNamara, B. y Hegney, D. Who Needs Bereavement Support? A Population Based Survey of Bereavement Risk and Support Need. PLOS ONE [Internet]. 26 de marzo de 2015 [citado 17 de febrero de 2020]; 10(3): e0121101. Disponible en https://journals.plos.org/plosone/article?id=10.1371/ journal.pone.0121101.

${ }^{16}$ Ferré-Grau, C., Sevilla-Casado, M., Boqué-Cavallé, M., Aparicio-Casals, M.R., Valdivieso-López, A. y Lleixá-Fortuño, M. Efectividad de la técnica de resolución de problemas aplicada por enfermeras: disminución de la ansiedad y la depresión en cuidadoras familiares. Aten Primaria [Internet]. 1 de diciembre de 2012 [citado 9 de febrero de 2020]; 44(12):695-701. Disponible en http://www.elsevier.es/es-revista-atencion-primaria-27- articulo-efectividad-tecnica-resolucion-problemas-aplicada-S0212656712002089.

${ }_{17}$ YI, PY. Duelo: Factores de riesgo de duelo complicado en cuidados paliativos [Internet]. Universitat de València; 2016 [citado 10 de febrero de 2020]. Disponible en https://dialnet.unirioja. es/servlet/tesis?.codigo $=76429$.

${ }^{18}$ Ruiz-Robledillo N. y Moya-Albiol, L. El cuidado informal: una visión actual. Revista de. Motivación y Emoción. 2012, 1, 22-30. ISSN 2012, 1576-4214.

${ }^{19}$ Izquierdo, M.D. y Duarte-Clíments, G. El sistema invisible de los cuidados. En sobre el Morir y la Muerte. Ed. Alfonso García. 2002 803-825. 
dades de cuidado, recurren al cuidado formal ${ }^{20}$. La revisión liderada por Zabalegui, tras analizar más de 300 publicaciones, confirma que pese a que la familia algunas veces paga a una tercera persona para ayudarles con las actividades del cuidado, el soporte informal en Espańa tiene sus raíces en el sistema familiar, constituyendo la gran mayoría de los cuidadores ${ }^{21}$.

El cuidador familiar suele ser una mujer entre 45-69 años que presenta vulnerabilidad previa, lo que le hace más proclive a sufrir complicaciones. Así, presentan problemas de ansiedad, depresión ${ }^{22}$, angustia emocional ${ }^{23}$, sobrecarga ${ }^{24}$, bajo nivel de estudios, sin trabajo, bajo nivel socioeconómico ${ }^{25}$ y además lleva ocupándose de las necesidades de su familiar dependiente durante un largo periodo de tiempo ${ }^{26}$. Además, en el contexto de la enfermedad terminal, la cuidadora no suele estar preparada para la muerte de su ser querido ${ }^{27}$. El cuidado puede tener efectos perjudi-

${ }^{20}$ Emanuel, E.J., Slutsman, J., Alpert, H., Baldwin, D. y Emanuel, L.L. Assistance from Family Members, Friends, Paid Care Givers, and Volunteers in the Care of Terminally Ill Patients. N Engl J Med [Internet]. 23 de septiembre de 1999 [citado 9 de febrero de 2020]; 341(13): 956-63. Disponible en http://www.nejm.org/doi/abs/ 10.1056/NEJM199909233411306.

${ }^{21}$ Zabalegui, A., Rodríguez, E., Ramírez, A.M., Pulpón, A., López, L., Izquierdo, M.D., Gual, P., González-Valentín, A., Gallart, A., Díaz, M., Corrales, E., Cabrera, E. y Bover, A. Revisión de evidencias: cuidadores informales de personas mayores de 65 ańos. Evidentia 2007 ene-febrero; 4(13). En http://www.index-f.com/evidentia/n13/291articulo.php [ISSN: 1697-638X].

22 Ribas, J., Castel, A., Escalada, B., Ugas, L., Grau, C., Magarolas, L. et al. Trastornos psico- patológicos del cuidador principal no profesional de pacientes ancianos. Rev Psiquiatría Fac Med Barna 2000; 27: 131-134. Sánchez, R.M., Sancho, M.C.C., García, E.L.H., Pérez, M.M., Perera, M.L.G., Pérez, L.S., et al. Perfil del cuidador principal en el área de salud de Gran Canaria. Revista Ene de Enfermería [Internet]. 21 de agosto de 2014 [citado 10 de febrero de 2020]; 8(2). Disponible en http://ene-enfermeria.org/ojs/index.php/ENE/article/view/435.

${ }^{23}$ Cameron, J.I., Franche, R.-L., Cheung, A.M. y Stewart, D.E. Lifestyle interference and emotional distress in family caregivers of advanced cancer patients. Cancer. 15 de enero de 2002; 94(2): 521-527.

${ }^{24}$ López Gil, M.J., Orueta Sánchez, R., Gómez-Caro, S., Sánchez Oropesa, A., Carmona de la Morena, J., Alonso Moreno, F.J. El rol de Cuidador de personas dependientes y sus repercusiones sobre su Calidad de Vida y su Salud. Revista Clínica de Medicina de Familia [Internet]. junio de 2009 [citado 10 de febrero de 2020]; 2(7): 332-339. Disponible en http://scielo.isciii. es/scielo.php?script=sci_abstract\&pid=S1699-695-X2009000200004\&lng=es\&nrm=iso\&tlng=es.

${ }^{25}$ Martínez-Román, M.A. La sobrecarga de las cuidadoras de personas dependientes. Análisis y propuestas de intervención psicosocial. Autores: Stephanie Carretero Gómez, Jorge Garcés Ferrer, Francisco Ródenas Rigla y Vicente Sanjosé López. Editorial: Tirant lo Blanch, Colección Políticas de Bienestar Social, Valencia. Año: 2006. 444 pp. ISBN: 84-8456-554-8. Alternativas: Cuadernos de trabajo social [Internet]. 2007 [citado 10 de febrero de 2020]; (15): 163. Disponible en https://dialnet.unirioja.es/ser- vlet/articulo?codigo $=5467138$.

${ }^{26}$ VÁzQuez Martínez, A. Salud del cuidador familiar de personas en situación de dependencia: modelo explicativo. 2014 [citado 10 de febrero de 2020]; Disponible en http://roderic. uv.es/handle/10550/36777.

${ }^{27}$ Hebert, R.S., Schulz, R., Copeland, V.C., Arnold, R.M. Preparing Family Caregivers for Death and Bereavement. Insights from Caregivers of Terminally Ill Patients. Journal of Pain and Symptom Management [Internet]. enero de 2009 [citado 10 de febrero de 2020]; 37(1): 3-12. Disponible en https://linkinghub.elsevier.com/retrieve/pii/ S0885392408002236. 
ciales en la salud física, psicológica y social de un cuidador y puede reducir significativamente su calidad de vida ${ }^{28}$. Es importante tener en cuenta que sus necesidades no están del todo satisfechas ${ }^{29}$. Se sabe poco sobre el ajuste a largo plazo de los cuidadores en el duelo ${ }^{30}$, su calidad de vida, bienestar y la mejor forma de proporcionar cuidados enfermeros eficaces a esta población ${ }^{31}$, pero parece que los sistemas actuales de apoyo no son adecuados para satisfacer esas necesidades. Se necesitan de manera urgente ensayos rigurosos con evaluaciones de proceso y descripciones de las intervenciones a realizar con los cuidadores ${ }^{32}$.

Respecto al cambio de tendencia diagnóstica en los periodos 2005-2010 y 2010-2014, puede deberse al cambio en la denominación en los diagnósticos, al pasar de Duelo Anticipado a Duelo. Parece más sencillo y fácilmente identificable un doliente normal que un deudo con duelo anticipado. Algunos estudios, como el realizado por Costello con ancianos que vivieron cómo su pareja recibía atención terminal en el hospital, muestran que estos cuidadores experimentan el duelo mientras cuidan del moribundo, produciendo este Duelo Anticipado un acercamiento en la relación que propicia mejor resolución de la aflicción ${ }^{33}$. El duelo anticipado con dependencia afectiva, educación más baja y el pobre apoyo específico al duelo, se manifiesta con incapacidad de dar sentido a la pérdida ${ }^{34}$. Cuando los cuidadores saben que sus familiares se dirigen hacia la muerte y son conscientes de la discapacidad y el sufrimiento del paciente, sufren por la pérdida del paciente antes de

28 Stenberg, U., Ruland, C.M., Miaskowski, C. Review of the literature on the effects of caring for a patient with cancer. Psycho-Oncology [Internet]. 2010 [citado 17 de febrero de 2020]; 19(10): 1013-25. Disponible en https://onlinelibrary.wiley.com/doi/abs/10.1002/pon.1670.

${ }^{29}$ Soothill, K., Morris, S.M., Thomas, C., Harman, J.C., Francis, B., McIllmuRRAY, M.B. The uni- versal, situational, and personal needs of cancer patients and their main carers. Eur J Oncol Nurs, marzo de 2003; 7(1): 5-13; discussion 14-16.

${ }^{30}$ Kim, Y., Carver, C.S., Spiegel, D., Mitchell, H.-R., Cannady, R.S. Role of family caregivers' self-perceived preparedness for the death of the cancer patient in long-term adjustment to bereavement. Psychooncology. 2017; 26(4): 484-492.

31 Holtslander, L.F. Caring for Bereaved Family Caregivers: Analyzing the Context of Care. Clinical Journal of Oncology Nursing [Internet]. 1 de junio de 2008 [citado 10 de febrero de 2020]; 12(3): 501-506. Disponible en http://cjon.ons.org/cjon/12/3/caring-be- reaved-family-caregivers-analyzing-context-care.

32 Treanor, C.J., Santin, O., Prue, G., Coleman, H., Cardwell, C.R., O’HalloRan, P. y Donnelly, M. Psychosocial interventions for informal caregivers of people living with cancer. Cochrane Database of Systematic Reviews 2019, Issue 6. Art. No.: CD009912. DOI: 10.1002/14651858.CD009912.pub2.

33 Anticipatory grief: coping with the impending death of a partner| International Journal of Palliative Nursing [Internet]. [citado 10 de febrero de 2020]. Disponible en https://www.magonlinelibrary.com/doi/abs/10.12968/ijpn.1999.5.5.8962.

${ }^{34}$ Burke, L.A., Clark, K.A., Ali, K.S., Gibson, B.W., Smigelsky, M.A. y Neimeyer, R.A. Risk Factors for Anticipatory Grief in Family Members of Terminally Ill Veterans Receiving Palliative Care Services. Journal of Social Work in End-of-Life \& Palliative Care [Internet]. 2 de octubre de 2015 [citado 10 de febrero de 2020]; 11(3-4): 244-266. Disponible en http://www.tandfonline.com/doi/full/10.1080/15524256.2015.1110071. 
la muerte, es decir, el duelo afecta a muchos cuidadores antes de la pérdida real ${ }^{35}$. Desde el diagnóstico hasta el fallecimiento, el cuidador padece las consecuencias biomédicas, psicosociales de la enfermedad y la percepción de una muerte inevitable, y experimenta sufrimiento e impacto emocional negativo ${ }^{36}$, especialmente en determinados procesos ${ }^{37}$. Esta afectación presenta diferencias según si el cuidado se ejerce sobre adultos jóvenes o adolescentes ${ }^{38}$.

Los Patrones Funcionales, adoptados por el Servicio Canario de Salud en la historia clínica electrónica de Atención Primaria, son una herramienta útil, aunque insuficiente, que requiere de un adecuado desarrollo teórico. No se debe perder de vista que la elección de este marco de valoración elegida deja todo abierto a la consideración del profesional a la hora de valorar en el duelo los síntomas de separación, las cogniciones, las conductas, la búsqueda de significados y las lesiones de la pérdida. Resulta paradójico que en casi la mitad de las personas diagnosticadas de Duelo Complicado la enfermera registrara una valoración de dicho patrón con un resultado de Normal o Riesgo de Alteración del Patrón. Además, los duelos no complicados presentaban peores resultados en la valoración previa. Es evidente la debilidad de la valoración propuesta por el Servicio Canario de Salud para los problemas psicosociales. Esta herramienta supone una división artificial de un todo integral. Se hace difícil establecer conexiones que lleven a la identificación de un fenómeno multidimensional, subjetivo y complejo como el duelo, «que incluye normas, expectativas, posición en la sociedad, ideologías religiosas o estéticas, posicionamiento ante el mundo, etc. y permite aludir a dimensiones positivas y negativas del mismo ${ }^{39}$.

35 Schulz, R., O’Brien, A.T, Bookwala, J., Fleissner, K. Psychiatric and physical morbidity effects of dementia caregiving: prevalence, correlates, and causes. Gerontologist, diciembre de 1995; 35(6): 771-791.

36 Limonero, J.T., Maté, J., Mateo, D., González-Barboteo, J., Bayés, R., BerNaus, M., et al. Desarrollo de la escala DME-C: una escala para la detección del malestar emocional de los cuidadores principales de personas con enfermedad avanzada o al final de la vida. Ansiedad y Estrés [Internet]. julio de 2016 [citado 10 de febrero de 2020]; 22(2-3): 104-9. Disponible en https://linkinghub.elsevier.com/retrieve/pii/S1134793716300288.

37 YANG, B.-H., Mu, P.-F. y WANG, W.-S. The experiences of families living with the anticipa- tory loss of a school-age child with spinal muscular atrophy - the parents' perspecti- ves. J Clin Nurs [Internet], septiembre de 2016 [citado 10 de febrero de 2020]; 25(17-18): 2648-57. Disponible en http://doi.wiley.com/10.1111/jocn.13312.

${ }^{38}$ Griffith, R., Davies, K., y Lavender, V. The characteristics and experiences of anticipatory mourning in caregivers of teenagers and young adults. International Journal of Palliati- ve Nursing [Internet]. 2 de noviembre de 2015 [citado 10 de febrero de 2020]; 21(11): 527-533. Disponible en http://www.magonlinelibrary.com/doi/10.12968/ijpn. 2015.21.11.527.

${ }^{39}$ García, A. Vivir el duelo. La experiencia de perder un hijo | Academia.edu [Internet]. [citado 10 de febrero de 2020]. Disponible en https://www.academia.edu/35322110/Vivir_el_duelo._ La_experiencia_de_perder_un_hijo. Aranda, S. y Milne, D. Guidelines for the assessment of complicated bereavement risk in family members of people receiving palliative care. Melbourne: Centre for Palliative Care. 2000. 
Aunque se recomienda el juicio clínico del profesional, la utilización de un instrumento de valoración, validado y estandarizado, redunda en la prestación de unos cuidados de mayor calidad. La validación de herramientas eficaces de valoración enfocadas a detectar estos problemas y, por ende, una determinada necesidad de cuidados es básica. El desarrollo de instrumentos de recolección de datos fiables para la práctica enfermera mediante estudios clínicos con el diseño adecuado, sobre todo de características definitorias ya descritas, resulta fundamental para el desarrollo de la ciencia del cuidado ${ }^{40}$.

En la pérdida, la valoración del riesgo debe realizarse evaluando los factores de riesgo y utilizando el juicio clínico y la información acerca del entorno familiar. En esta línea, existen herramientas validadas en nuestro entorno como el Cuestionario para el Diagnóstico enfermero psicosocial, CdePS, que facilita el enunciado de hasta 28 etiquetas diagnósticas psicosociales agrupadas en seis dimensiones (percepción-manejo de la salud, conducta-interacción social, emocional-autopercepción, cuidador-sobrecarga, pérdida-duelo, trastorno de imagen $)^{41}$. La ambigüedad de las características definitorias asociadas a cada uno de los diagnósticos enfermeros de Duelo hace difícil construir con ellas una herramienta de valoración que delimite cuándo un proceso es normal, complicado o de riesgo. Las personas establecen vínculos emocionales a lo largo de su vida y, cuando estos vínculos se rompen, sufren el dolor por la pérdida y, por tanto, el proceso de duelo ${ }^{42}$.

En España, existen propuestas de valoración por patrones funcionales de salud más amplias que la propuesta por el Servicio Canario de Salud. Por ejemplo, el proyecto de Conocimiento Enfermero Estandarizado (CENES), proyecto de investigación taxonómica del Departamento de Metodología Enfermera de la Fundación para el Desarrollo de Enfermería que pretende «facilitar la aplicación en la práctica profesional de los cuidados enfermeros y permitir su inclusión en los sistemas de expertos de información, facilitando la evaluación e investigación en este campo »33, en la valoración del octavo patrón pregunta específicamente por la pérdida a través de los ítems: Pérdida de Seres queridos; Conductas inefectivas en el duelo; Expre-

${ }^{40}$ Lunney, M. The Need for International Nursing Diagnosis Research and a Theoretical Framework. International Journal of Nursing Terminologies and Classifications [Inter- net]. 2008 [citado 21 de febrero de 2020]; 19(1): 28-34. Disponible en https://onlineli- brary.wiley.com/ doi/abs/10.1111/j.1744-618X.2007.00076.x. The Need for International Nursing Diagnosis Research and a Theoretical Framework -Lunney -2008- International Journal of Nursing Terminologies and Classifications-Wiley Online Library [Internet]. [citado 21 de febrero de 2020]. Disponible en https:// onlinelibrary.wiley.com/doi/abs/10.1111/j.1744-618X.2007.00076.x.

${ }_{41}$ Brito, P.R. y Aguirre, A. El cuestionario para el diagnóstico enfermero psicosocial (CdePS). Diagnóstico de problemas y síndromes psicosociales. En: Brito, P.R., Aguirre, A. El diagnóstico psicosocial desde una perspectiva enfermera. Barcelona: Elsevier, 50-67. 2014.

42 Garcia, A.M. El duelo. Un espacio intermedio de aprendizaje en la vida. Editor Bubok Publishing S.L. España. ISBN papel: 978-84-686-2218-7; ISBN ebook: 978-84-686-2219-4. 2012.

43 Arribas, A.A., Aréjula, J.L., Borrego, R., Domingo, M., Morente, M., Robledo, J. y Santamaría, J.M. Valoración Enfermera Estandarizada. Clasificación de los criterios de valoración de enfermería. ISBN 84-89174-96-2. FUDEN. Observatorio de Metodología Enfermera. 2006. 
siones de culpa; Negación de una pérdida; Duelo Anticipado. Pese a ser más completa, queda muy lejos respecto a una valoración global del duelo.

Aunque las guías de práctica clínica indican la necesidad de nuevos estudios que valoren su validez, en la literatura especializada encontramos diferentes escalas y herramientas. Por ejemplo, el Invetory of complicated Grief propuesto por Prigerson $^{44}$ tras estudiar a 97 ancianos viudos, un instrumento sencillo y de los más citados en la literatura especializada o instrumentos como el Inventario de Experiencias en Duelo (IED) de Catherine Sanders ${ }^{45}$; el Cuestionario de Riesgo de Duelo Complicado (CRDC) de Parkes y Weiss ${ }^{46}$; el Inventario de Texas revisado de Faschingbauer $^{47} \mathrm{o}$ el Grief and Meaning Reconstruction Inventory propuesto por Gillies ${ }^{48}$. Por otra parte, existe una correlación entre la intensidad de síntomas con el número total de pérdidas de ser querido no superadas y con las pérdidas no superadas de otra índole ${ }^{49}$, por lo que es necesario explorar la historia previa de pérdidas ${ }^{50}$.

Esta investigación muestra diferencias en los antecedentes médicos relacionados con depresión y ansiedad en los dolientes, lo que coincide con estudios previos. Respecto a la ansiedad, se aprecian diferencias respecto a los duelos calificados como de riesgo y los normales. Además, se evidencian grandes diferencias por género en ambos problemas. Canarias se sitúa como la mayor Comunidad Autónoma con problemas declarados relacionados con Ansiedad y Depresión, con un

${ }^{4}$ Prigerson, H.G., Maciejewsimi, P.K., Reynolds, C.F., Bierhals, A.J., Newsom, J.T., FAsiczKa, A., et al. Inventory of complicated grief: A scale to measure maladaptive symptoms of loss. Psychiatry Research [Internet]. 29 de noviembre de 1995 [citado 2 de marzo de 2020]; 59(1): 65-79. Disponible en http://www.sciencedirect.com/science/article/pii/ 0165178195027572. García, J.T.L., Reverte, M.A.L., García, J.A.G., Méndez, J.M. y Prigerson, H.G. Adaptación al castellano del inventario de duelo complicado. Medicina paliativa [Internet]. 2009 [citado 2 de marzo de 2020]; 16(5): 291-297. Disponible en https://dialnet.unirioja.es/ser-vlet/articulo?codigo=3068410.

${ }^{45}$ SAnders, C.M. Typologies and symptoms of adult bereavement [disertación]. Tampa: University of South Florida. 1977. García-García, J.A., Landa Petralanda, V., Trigueros ManZANO, M.C. y GAMINDE INDA, I. Inventario de experiencias en duelo (IED): adaptación al castellano, fiabilidad y validez. Atención Primaria [Internet]. 1 de enero de 2001 [citado 2 de marzo de 2020]; 27(2): 86-93. Disponible en http://www.sciencedirect.com/science/article/pii/S0212656701787793.

46 Parke, C.M., Weiss RS. Recovery from bereavement. New York: Basic Books. 1983.

${ }^{47}$ García García, J.A., Landa Petralanda, V., Trigueros Manzano, M.C., Gaminde INDA, I. Inventario Texas Revisado de Duelo (ITRD): adaptación al castellano, fiabilidad y validez. Aten Primaria [Internet]. 30 de abril de 2005 [citado 2 de marzo de 2020];35(7): 353-8. Disponible en http://www.elsevier.es/es-revista-atencion-primaria-27-articulo-inventa- rio-texas-revisado-duelo-itrd--13074293.

${ }^{48}$ Gillies, J.M., Neimeyer, R.A. y Milman, E. The Grief and Meaning Reconstruction Inven- tory (GMRI): Initial Validation of a New Measure. Death Studies [Internet]. 7 de febrero de 2015 [citado 2 de marzo de 2020]; 39(2): 61-74. Disponible en https://doi.org/10.1080/0748118 7.2014.907089.

${ }^{49}$ Chaurand, A., Feixa, G., Neimeyer, R.A., Salla, M. y Trujillo, A. History of losses and de- pressive symptoms. Revista Argentina de Clinica Psicologica. August 2015.

50 Viaplana, G.F., Neimeyer, R.A. y Chaurand, A. El inventario de historia de pérdidas (IHP): presentación y utilidad clínica. Revista de psicoterapia [Internet]. 2010 [citado 2 de marzo de 2020]; 21(84): 95-101. Disponible en https://dialnet.unirioja.es/servlet/articu- lo?codigo=3740176. 
20,58\%, siendo la media estatal es $15,03 \%{ }^{51}$. El estudio de Díaz-Cruz acerca de la prevalencia de los trastornos mentales en la isla de Tenerife estima, tras utilizar entrevistas estructuradas y el cuestionario de salud de Goldberg, que la prevalencia estimada de Trastornos de ansiedad en esta población es $14,5 \% \%^{52}$. El proyecto ESEMed-España, que tiene como objetivo principal obtener datos sobre los trastornos mentales en Espańa, muestra que la prevalencia en España de trastorno de depresión mayor es menor que en otros países occidentales. La prevalencia de por vida es de 10,6\%, mientras que la prevalencia de 12 meses es de 4\%. Además, el hecho de ser mujer aumenta el riesgo ${ }^{53}$. La revisión y liderada por Steel, que incluye los estudios publicados entre 1980 y 2013, confirma que las mujeres experimentan mayores tasas de prevalencia de trastornos de ánimo y ansiedad ${ }^{54}$, lo que coincide con el presente estudio respecto a los diagnósticos médicos relacionados con la ansiedad y depresión y la etiqueta diagnóstica enfermera Ansiedad. Además, se aprecian diferencias en otras etiquetas que parecen indicar que la pérdida produce un impacto diferente en hombres y mujeres, no sólo por su estilo de afrontamiento de la pérdida en concreto, también porque previamente presenten diferentes problemas y necesidades de cuidados que pueden incidir en el desarrollo del duelo.

Sólo en las etiquetas diagnósticas Riesgo de Traumatismo y Riesgo de Soledad se hallan diferencias significativas a favor de Duelo Complicado. Es decir, en los dolientes con complicaciones no se habían detectado más necesidades de cuidado detectadas previas a la pérdida. La regresión logística desarrollada en este estudio mostró que, en lo que a etiquetas diagnósticas enfermeras se refiere, únicamente Riesgo de Soledad se comportó como un factor predictor de complicaciones en el duelo. La soledad, junto con la muerte, la libertad y la falta de significado forma, los cuatro conflictos básicos de la existencia según Yalom ${ }^{55}$. Esta etiqueta diagnóstica está definida en la clasificación de diagnósticos de enfermería NANDA, como «vulnerable a experimentar malestar asociado al deseo o necesidad de un mayor contacto con los demás, que puede comprometer la salud», teniendo como factores de riesgo ais-

51 Ministerio de Sanidad, Servicios Sociales e Igualdad. Indicadores de Salud 2013. Evolución de los indicadores del estado de salud en España y su magnitud en el contexto de la Unión Europea. Madrid: Ministerio de Sanidad, Servicios Sociales e Igualdad, 2014.

52 Díaz-Cruz, F., Bethencourt Pérez, J.M. y Peñate Castro, W. Prevalencia de los trastornos mentales en la isla de Tenerife. Revista de la Asociación Española de Neuropsiquiatría [Internet]. junio de 2004 [citado 10 de febrero de 2020]; (90): 21-39. Disponible en http://scielo.isciii.es/ scielo.php?script=sci_abstract\&pid=S0211-57352004000200003\&lng=es\&nrm=iso\&tlng=es.

53 Gabilondo, A., Rojas-Farreras, S., Vilagut, G., Haro, J.M., Fernández, A., PinTo-MezA, A., et al. Epidemiology of major depressive episode in a southern European country: Results from the ESEMeD-Spain project. Journal of Affective Disorders [Internet], enero de 2010 [citado 10 de febrero de 2020]; 120(1-3): 76-85. Disponible en https://linking- hub.elsevier.com/ retrieve/pii/S016503270900161X.

54 Steel, Z., Marnane, C., Iranpour, C., Chey, T., Jackson, J.W., Patel, V., et al. The global pre- valence of common mental disorders: a systematic review and meta-analysis 19802013. International Journal of Epidemiology [Internet]. abril de 2014 [citado 10 de febrero de 2020]; 43(2): 476-493. Disponible en https://academic.oup.com/ije/article-loo-kup/doi/10.1093/ije/dyu038.

55 Yalom, I. Psicoterapia Existencial, Tr. Diorki. Barcelona: Herder, 1a edición, 1984. 
lamiento físico, aislamiento social, deprivación afectiva y deprivación emocional ${ }^{56}$. La relación entre la prolongación de la soledad y duelo con complicaciones ya está planteada en la bilbiografía ${ }^{57}$. La detección de la soledad y el aislamiento social por las enfermeras es importante $e^{58}$ y existen instrumentos de valoración multidisciplinares que han demostrado ser eficaces ${ }^{59}$. Santos, en un estudio de ancianos institucionalizados en Brasil, relaciona la presencia de este diagnóstico con la depresión, proponiendo una serie de intervenciones y acciones enfermeras ${ }^{60}$.

Esta investigación no está exenta de limitaciones. En primer lugar, abordar un fenómeno como el duelo desde una óptica estrictamente cuantitativa y de forma retrospectiva. En segundo, las limitaciones propias de las etiquetas diagnósticas a estudio, que están siendo actualmente revisadas por el comité de desarrollo diagnóstico de NANDA-I.

\section{CONCLUSIÓN}

El duelo es un fenómeno multidimensional que difícilmente puede ser valorado desde una herramienta reduccionista. Se debe profundizar en la alta prevalencia de complicaciones en el duelo con la mujer, asociándolo a las cargas de cuidado y vulnerabilidad previa. La asociación entre la soledad y el duelo complicado es evidente y el papel de las enfermeras comunitarias en estos pacientes puede ser clave. Es necesario seguir investigando acerca de las necesidades de cuidados en los dolientes, con y sin complicaciones, así como en sus factores predictores y protectores.

${ }^{56}$ Herdman, T.H. y Kamitsuru, S. (eds.). NANDA International Nursing Diagnoses: Defini- tions \& Classification, 2015-2017. Oxford: Wiley Blackwell, 2014.

${ }^{57}$ Large, T. Some Aspects of Loneliness in Families. Family Process [Internet]. 1989 [citado 12 de febrero de 2020]; 28(1): 25-35. Disponible en https://onlinelibrary.wiley.-com/doi/ abs/10.1111/j.1545-5300.1989.00025.x.

${ }^{58}$ McInnis, G.J., White, J.H. A phenomenological exploration of loneliness in the older adult. Arch Psychiatr Nurs. junio de 2001; 15(3): 128-139.

59 Puig Llobet, M., Lluch Canut, M.T., Rodríguez Ávila, N. Valoración de enfermería: detección de la soledad y del aislamiento social en los ancianos de riesgo. Gerokomos [Internet]. marzo de 2009 [citado 12 de febrero de 2020]; 20(1): 9-14. Disponible en http://scielo.isciii. es/scielo.php?script=sci_abstract\&pid=S1134-928X2009000100002\&lng=es\&nrm=iso\&tlng=es.

${ }^{60}$ Santos, S.S.C., Tier, C.G., Silva, B.T., Barlem, E.L.D., Felicianni, A.M. y ValcaRENGHI, F.V. Diagnósticos e intervenciones de enfermería para ancianos con depresión y residentes en una institución de larga estancia (ILE). Enfermería Global [Internet]. octubre de 2010 [citado 12 de febrero de 2020]; (20): 0-0. Disponible en http://scielo.isciii.es/scielo.php?script=sci_abstract\&pi$\mathrm{d}=$ S1695-61412010000300003\&lng=es\&nrm=iso\&tlng=es. 


\section{AGRADECIMIENTOS}

A la Dirección General de Programas Asistenciales del Servicio Canario de Salud por su colaboración en la explotación de datos.

\section{CONFLICTO DE INTERESES}

El autor declara no tener conflicto de intereses.

RECIBIDO: 28-11-2019; ACEPTADO: 2-2-2020 


\section{BIBLIOGRAFÍA}

Alfaro-LeFevre, R. Aplicacion Del Proceso Enfermero Fomentar el Cuidado en Colaboracion. Elsevier España; 2003. 308 p.

Anticipatory grief: coping with the impending death of a partner| International Journal of Palliative Nursing [Internet]. [citado 10 de febrero de 2020]. Disponible en https://www.magonlinelibrary.com/doi/abs/10.12968/ijpn.1999.5.5.8962.

Aoun, S.M., Breen, L.J., Howting, D.A., Rumbold, B., McNamara, B. y Hegney, D. Who Needs Bereavement Support? A Population Based Survey of Bereavement Risk and Support Need. PLOS ONE [Internet]. 26 de marzo de 2015 [citado 17 de febrero de 2020]; 10(3): e0121101. Disponible en https://journals.plos.org/plosone/article?id=10.1371/ journal.pone.0121101.

Aranda, S. y Milne, D. Guidelines for the assessment of complicated bereavement risk in family members of people receiving palliative care. Melbourne: Centre for Palliative Care. 2000.

Arribas, A.A., Aréjula, J.L., Borrego, R., Domingo, M., Morente, M., Robledo, J. y SantaMARÍA, J.M. Valoración Enfermera Estandarizada. Clasificación de los criterios de valoración de enfermería. ISBN 84-89174-96-2. FUDEN. Observatorio de Metodología Enfermera. 2006.

Brito P.R., Aguirre A., Duarte G. y Sánchez, M.B. «Investigación epidemiológica en diagnósticos enfermeros», en Echevarría, P. Investigación en metodología y lenguajes en enfermeros. Barcelona: Elsevier, 187-199.

Brito, P.R. y Aguirre, A. «El cuestionario para el diagnóstico enfermero psicosocial (CdePS). Diagnóstico de problemas y síndromes psicosociales», en: Brito, P.R., Aguirre, A. El diagnóstico psicosocial desde una perspectiva enfermera. Barcelona: Elsevier, 50-67. 2014.

Burke, L.A., Clark, K.A., Ali, K.S., Gibson, B.W., Smigelsky, M.A., Neimeyer, R.A. «Risk Factors for Anticipatory Grief in Family Members of Terminally Ill Veterans Receiving Palliative Care Services». Journal of Social Work in End-of-Life \& Palliative Care [Internet]. 2 de octubre de 2015 [citado 10 de febrero de 2020]; 11(3-4):244-66. Disponible en http:// www.tandfonline.com/doi/full/10.1080/15524256.2015.1110071.

Cameron, J.I., Franche, R.-L., Cheung, A.M. y Stewart, D.E. Lifestyle interference and emotional distress in family caregivers of advanced cancer patients. Cancer. 15 de enero de 2002; 94(2): 521-7.

Carpenito L.J. Diagnóstico de Enfermería. 15. ${ }^{a}$ Edición. Interamericana-McGraw-Hill. 2017.

Chaurand, A., Feixa, G., Neimeyer, R.A., Salla, M. y Trujillo, A. History of losses and depressive symptoms. Revista Argentina de Clinica Psicologica. August 2015.

Díaz-Cruz, F., Bethencourt Pérez, J.M. y Peñate Castro, W. «Prevalencia de los trastornos mentales en la isla de Tenerife». Revista de la Asociación Española de Neuropsiquiatría [Internet]. junio de 2004 [citado 10 de febrero de 2020]; (90):21-39. Disponible en http://scielo. isciii.es/scielo.php?script=sci_abstract\&pid=S0211-57352004000200003\&lng=es\&nr$\mathrm{m}=\mathrm{iso} \& \mathrm{t} \operatorname{lng}=\mathrm{es}$.

Emanuel, E.J., Slutsman, J., Alpert, H., Baldwin, D. y Emanuel, L.L. Assistance from Family Members, Friends, Paid Care Givers, and Volunteers in the Care of Terminally Ill Patients. N Engl J Med [Internet]. 23 de septiembre de 1999 [citado 9 de febrero de 2020]; 341(13): 956-963. Disponible en http://www.nejm.org/doi/abs/ 10.1056/NEJM199909233411306. 
Ferré-Grau, C., Sevilla-Casado, M., Boqué-Cavallé, M., Aparicio-Casals, M.R., Valdivieso-López, A. y Lleixá-Fortuño, M. Efectividad de la técnica de resolución de problemas aplicada por enfermeras: disminución de la ansiedad y la depresión en cuidadoras familiares. Aten Primaria [Internet]. 1 de diciembre de 2012 [citado 9 de febrero de 2020]; 44(12): 695-701. Disponible en http://www.elsevier.es/es-revista-atencion-primaria-27- articulo-efectividad-tecnica-resolucion-problemas-aplicada-S0212656712002089.

Gabilondo, A., Rojas-Farreras, S., Vilagut, G., Haro, J.M., Fernández, A., Pinto-Meza, A., et al. Epidemiology of major depressive episode in a southern European country: Results from the ESEMeD-Spain project. Journal of Affective Disorders [Internet], enero de 2010 [citado 10 de febrero de 2020]; 120(1-3): 76-85. Disponible en https://linking- hub.elsevier.com/retrieve/pii/S016503270900161X.

García García, J.A., Landa Petralanda, V., Trigueros Manzano, M.C., Gaminde Inda, I. Inventario Texas Revisado de Duelo (ITRD): adaptación al castellano, fiabilidad y validez. Aten Primaria [Internet]. 30 de abril de 2005 [citado 2 de marzo de 2020];35(7): 3538. Disponible en http://www.elsevier.es/es-revista-atencion-primaria-27-articulo-inventario-texas-revisado-duelo-itrd--13074293.

García, A. Vivir el duelo. La experiencia de perder un hijo | Academia.edu [Internet]. [citado $10 \mathrm{de}$ febrero de 2020]. Disponible en https://www.academia.edu/35322110/Vivir_el_duelo._ La_experiencia_de_perder_un_hijo.

García, A.M. El duelo. Un espacio intermedio de aprendizaje en la vida. Editor Bubok Publishing S.L. España. ISBN papel: 978-84-686-2218-7; ISBN ebook: 978-84-686-2219-4. 2012.

García, J.T.L., Reverte, M.A.L., García, J.A.G., Méndez, J.M. y Prigerson, H.G. Adaptación al castellano del inventario de duelo complicado. Medicina paliativa [Internet]. 2009 [citado 2 de marzo de 2020]; 16(5): 291-7. Disponible en https://dialnet.unirioja.es/servlet/articulo?codigo $=3068410$.

García-García, J.A., Landa Petralanda, V., Trigueros Manzano, M.C. y Gaminde Inda, I. Inventario de experiencias en duelo (IED): adaptación al castellano, fiabilidad y validez. Atención Primaria [Internet]. 1 de enero de 2001 [citado 2 de marzo de 2020]; 27(2): 86-93. Disponible en http://www.sciencedirect.com/science/article/pii/S0212656701787793.

Gillies, J.M., Neimeyer, R.A. y Milman, E. The Grief and Meaning Reconstruction Inven- tory (GMRI): Initial Validation of a New Measure. Death Studies [Internet]. 7 de febrero de 2015 [citado 2 de marzo de 2020]; 39(2): 61-74. Disponible en https://doi.org/10.1080/0 7481187.2014.907089.

Gordon, M. Manual of Nursing Diagnosis, 1988-89: Including All Diagnostic Categories Approved by the North American Nursing Diagnosis Association. C.V. Mosby; 1989. 378 p.

Griffith, R., Davies, K., y Lavender, V. The characteristics and experiences of anticipatory mourning in caregivers of teenagers and young adults. International Journal of Palliative Nursing [Internet]. 2 de noviembre de 2015 [citado 10 de febrero de 2020]; 21(11): 527533. Disponible en http://www.magonlinelibrary.com/doi/10.12968/ijpn. 2015.21.11.527.

Hebert, R.S., Schulz, R., Copeland, V.C., Arnold, R.M. Preparing Family Caregivers for Death and Bereavement. Insights from Caregivers of Terminally Ill Patients. Journal of Pain and Symptom Management [Internet]. enero de 2009 [citado 10 de febrero de 2020]; 37(1): 3-12. Disponible en https://linkinghub.elsevier.com/retrieve/pii/ S0885392408002236.

Herdman, T.H. y Kamitsuru, S. (eds.). NANDA International Nursing Diagnoses: Definitions \& Classification, 2018-2020. Oxford: Wiley Blackwell. 2017. 
Herdman, T.H. y Kamitsuru, S. (eds.). NANDA International Nursing Diagnoses: Defini- tions \& Classification, 2015-2017. Oxford: Wiley Blackwell, 2014.

Hogan, Nancy S., Worden, J. William y Schmidt, Lee A. An Empirical Study of the Proposed Complicated Grief Disorder Criteria-2004 [Internet]. [citado 14 de febrero de 2020]. Disponible en https://journals.sagepub.com/doi/abs/10.2190/GX7H-H05N- A4DN-RLU9?journalCode=omea.

Holtslander, L.F. Caring for Bereaved Family Caregivers: Analyzing the Context of Care. Clinical Journal of Oncology Nursing [Internet]. 1 de junio de 2008 [citado 10 de febrero de 2020]; 12(3): 501-506. Disponible en http://cjon.ons.org/cjon/12/3/caring-be- reaved-family-caregivers-analyzing-context-care.

Izquierdo, M.D. y Duarte-Clíments, G. El sistema invisible de los cuidados. En sobre el Morir y la Muerte. Ed. Alfonso García. 2002 803-825.

Kim, Y., Carver, C.S., Spiegel, D., Mitchell, H.-R., Cannady, R.S. Role of family caregivers' self-perceived preparedness for the death of the cancer patient in long-term adjustment to bereavement. Psychooncology. 2017 26(4): 484-492.

Large, T. Some Aspects of Loneliness in Families. Family Process [Internet]. 1989 [citado 12 de febrero de 2020]; 28(1): 25-35. Disponible en https://onlinelibrary.wiley.-com/doi/abs/10.1111/ j.1545-5300.1989.00025.x.

Limonero, J.T., Maté, J., Mateo, D., González-Barboteo, J., Bayés, R., Bernaus, M., et al. Desarrollo de la escala DME-C: una escala para la detección del malestar emocional de los cuidadores principales de personas con enfermedad avanzada o al final de la vida. Ansiedad y Estrés [Internet]. julio de 2016 [citado 10 de febrero de 2020]; 22(2-3): 104-9. Disponible en https://linkinghub.elsevier.com/retrieve/pii/S1134793716300288.

López Gil, M.J., Orueta Sánchez, R., Gómez-Caro, S., Sánchez Oropesa, A., Carmona de la Morena, J., Alonso Moreno, F.J. El rol de Cuidador de personas dependientes y sus repercusiones sobre su Calidad de Vida y su Salud. Revista Clínica de Medicina de Familia [Internet]. junio de 2009 [citado 10 de febrero de 2020];2(7): 332-339. Disponible en http://scielo.isciii.es/scielo.php?script=sci_abstract\&pid=S1699-695-X2009000200004\&l$\underline{\mathrm{ng}=\mathrm{es} \& \mathrm{nrm}=\mathrm{iso} \& \mathrm{t} \operatorname{lng}=\mathrm{es}}$.

Lunney, M. The Need for International Nursing Diagnosis Research and a Theoretical Framework. International Journal of Nursing Terminologies and Classifications [Inter- net]. 2008 [citado 21 de febrero de 2020]; 19(1): 28-34. Disponible en https://onlineli- brary.wiley.com/doi/ abs/10.1111/j.1744-618X.2007.00076.x.

Martínez-Román, M.A. La sobrecarga de las cuidadoras de personas dependientes. Análisis y propuestas de intervención psicosocial. Autores: Stephanie Carretero Gómez, Jorge Garcés Ferrer, Francisco Ródenas Rigla y Vicente Sanjosé López. Editorial: Tirant lo Blanch, Colección Políticas de Bienestar Social, Valencia. Año: 2006. 444 pp. ISBN: 84-8456554-8. Alternativas: Cuadernos de trabajo social [Internet]. 2007 [citado 10 de febrero de 2020]; (15): 163. Disponible en https://dialnet.unirioja.es/ser-vlet/articulo?codigo=5467138.

McInnis, G.J., White, J.H. A phenomenological exploration of loneliness in the older adult. Arch Psychiatr Nurs. junio de 2001; 15(3): 128-139.

Ministerio de Sanidad, Servicios Sociales e Igualdad. Indicadores de Salud 2013. Evolución de los indicadores del estado de salud en España y su magnitud en el contexto de la Unión Europea. Madrid: Ministerio de Sanidad, Servicios Sociales e Igualdad, 2014. 
Отт, $\mathrm{CH}$. The impact of complicated grief on mental and physical health at various points in the bereavement process. Death Stud, abril de 2003; 27(3): 249-272.

Parke, C.M., Weiss RS. Recovery from bereavement. New York: Basic Books. 1983.

Prigerson, H.G., Maciejewski, P.K., Reynolds, C.F., Bierhals, A.J., Newsom, J.T., Fasiczka, A., et al. Inventory of complicated grief: A scale to measure maladaptive symptoms of loss. Psychiatry Research [Internet]. 29 de noviembre de 1995 [citado 2 de marzo de 2020]; 59(1): 65-79. Disponible en http://www.sciencedirect.com/science/article/pii/ 0165178195027572.

Puig Llobet, M., Lluch Canut, M.T., Rodríguez Ávila, N. Valoración de enfermería: detección de la soledad y del aislamiento social en los ancianos de riesgo. Gerokomos [Internet]. marzo de 2009 [citado 12 de febrero de 2020]; 20(1):9-14. Disponible en http://scielo.isciii.es/scielo. php?script=sci_abstract\&pid=S1134-928X2009000100002\&lng=es\&nrm=iso\&tlng=es.

Ribas, J., Castel, A., Escalada, B., Ugas, L., Grau, C., Magarolas, L. et al. Trastornos psicopatológicos del cuidador principal no profesional de pacientes ancianos. Rev Psiquiatría Fac Med Barna 2000; 27: 131-134.

Rodríguez Álvaro, M., Brito Brito, P.R., García Hernández, A.M. y Fernández GutiéRREZ, D.Á. Etiquetas diagnósticas predictoras de duelo complicado. En XII Simposium Internacional AENTDE. Cádiz, 2018.

Rodríguez Alvaro, M., García Hernández, A.M. y Toledo Rosell, C. Hacia una visión constructivista del Duelo. Index de Enfermería [Internet]. septiembre de 2008 [citado 14 de febrero de 2020]; 17(3): 193-196. Disponible en http://scielo.isciii.es/scielo.php?script=sci_ abstract\&pid=S1132-12962008000300009\&lng=es\&nrm=iso\&tlng=es.

Rodríguez, M. Epidemiología enfermera del duelo en canarias. Universidad de La Laguna; 2017 [citado 9 de febrero de 2020]. Disponible en https://www.educacion.es/ teseo/mostrarRef. do? ref $=1454574$.

RodríGuEz-Álvaro, M. Impacto del duelo complicado. una lectura a través del lenguaje del cuidado. Revista Ene de Enfermería [Internet]. 4 de septiembre de 2019 [citado 9 de febrero de 2020]; 13(3). Disponible en http://www.e-ne-enfermeria.org/ojs/index.php/ENE/article/view/915.

Rodríguez-Álvaro, M., Brito-Brito, P.R., García-Hernández, A.M., Aguirre-Jaime, A., FernÁndeZ-Gutiérrez, D.A. The Grieving Nursing Diagnoses in the Primary Healthcare Set- ting: The Grieving Nursing Diagnoses in the Primary Healthcare Setting. Int J Nurs Terminol Knowledge, enero de 2019; 30(1): 34-42.

Rodríguez-Álvaro, M., García-Hernández, A.M. y Brito-Brito, P.R. Hacia un modelo enfermero para entender la pérdida y el duelo. Enfermería Clínica. noviembre de 2017;27(6): 397-398.

Rodríguez-Álvaro, M., García-Hernández, A.M. y Brito-Brito, P.R. Grieving is not a Nursing Diagnosis. Int J Nurs Knowl. 13 de febrero de 2020; [Epub ahead of print] DOI: 10.1111/2047-3095.12278.

Rodríguez-Álvaro, M., García-Hernández, A.M., Brito-Brito, P.R., Aguirre-Jaime, A., FernándeZ-Gutiérrez, D.Á. Intervenciones y criterios de resultado planificados por las enfermeras comunitarias en la atención al duelo en Canarias. Enfermería Clínica. julio de 2018;28(4): 240-6.

Ruiz-Robledillo N. y Moya-Albiol, L. El cuidado informal: una visión actual. Revista de. Motivación y Emoción. 2012, 1, 22-30. ISSN 2012, 1576-4214. 
Sánchez, R.M., Sancho, M.C.C., García, E.L.H., Pérez, M.M., Perera, M.L.G., Pérez, L.S., et al. «Perfil del cuidador principal en el área de salud de Gran Canaria». Revista Ene de Enfermería [Internet]. 21 de agosto de 2014 [citado 10 de febrero de 2020];8(2). Disponible en http://ene-enfermeria.org/ojs/index.php/ENE/article/view/435.

SANDERS, C.M. Typologies and symptoms of adult bereavement [disertación]. Tampa: University of South Florida. 1977.

Santos, S.S.C., Tier, C.G., Silva, B.T., Barlem, E.L.D., Felicianni, A.M. y Valcarenghi, F.V. Diagnósticos e intervenciones de enfermería para ancianos con depresión y residentes en una institución de larga estancia (ILE). Enfermería Global [Internet]. octubre de 2010 [citado 12 de febrero de 2020]; (20):0-0. Disponible en http://scielo.isciii.es/scielo.php?script=sci_ abstract\&pid=S1695-61412010000300003\&lng=es\&nrm=iso\&tlng=es.

Schulz, R., O’Brien, A.T, Bookwala, J., Fleissner, K. Psychiatric and physical morbidity effects of dementia caregiving: prevalence, correlates, and causes. Gerontologist, diciembre de 1995; 35(6): 771-791.

Soothill, K., Morris, S.M., Thomas, C., Harman, J.C., Francis, B., McIllmurray, M.B. The uni- versal, situational, and personal needs of cancer patients and their main carers. Eur J Oncol Nurs, marzo de 2003; 7(1): 5-13; discussion 14-16.

Steel, Z., Marnane, C., Iranpour, C., Chey, T., Jackson, J.W., Patel, V., et al. The global prevalence of common mental disorders: a systematic review and meta-analysis 1980-2013. International Journal of Epidemiology [Internet]. abril de 2014 [citado 10 de febrero de 2020]; 43(2): 476-93. Disponible en https://academic.oup.com/ije/article-loo-kup/ doi/10.1093/ije/dyu038.

Stenberg, U., Ruland, C.M., Miaskowski, C. Review of the literature on the effects of caring for a patient with cancer. Psycho-Oncology [Internet]. 2010 [citado 17 de febrero de 2020];19(10):1013-25. Disponible en https://onlinelibrary.wiley.com/doi/abs/10.1002/ pon. 1670 .

The Need for International Nursing Diagnosis Research and a Theoretical Framework -Lunney-2008International Journal of Nursing Terminologies and Classifications-Wiley Online Library [Internet]. [citado 21 de febrero de 2020]. Disponible en https://onlinelibrary.wiley.com/ doi/abs/10.1111/j.1744-618X.2007.00076.x.

Treanor, C.J., Santin, O., Prue, G., Coleman, H., Cardwell, C.R., O’Halloran, P. y DonNELLY, M. Psychosocial interventions for informal caregivers of people living with cancer. Cochrane Database of Systematic Reviews 2019, Issue 6. Art. No.: CD009912. DOI: 10.1002/14651858.CD009912.pub2.

VÁzquez Martínez, A. Salud del cuidador familiar de personas en situación de de- pendencia: modelo explicativo. 2014 [citado 10 de febrero de 2020]; Disponible en http://roderic. uv.es/handle/10550/36777.

Viaplana, G.F., Neimeyer, R.A. y Chaurand, A. El inventario de historia de pérdidas (IHP): presentación y utilidad clínica. Revista de psicoterapia [Internet]. 2010 [citado 2 de marzo de 2020];21(84):95-101. Disponible en https://dialnet.unirioja.es/servlet/articu- lo?co$\underline{\text { digo }=3740176 \text {. }}$.

Yalom, I. Psicoterapia Existencial, Tr. Diorki. Barcelona: Herder, 1. ${ }^{a}$ edición, 1984.

YANG, B.-H., Mu, P.-F. y WANG, W.-S. The experiences of families living with the anticipatory loss of a school-age child with spinal muscular atrophy-the parents' perspectives. J. Clin Nurs 
[Internet], septiembre de 2016 [citado 10 de febrero de 2020]; 25(17-18): 2648-57. Disponible en http://doi.wiley.com/10.1111/jocn.13312.

YI, PY. Duelo: Factores de riesgo de duelo complicado en cuidados paliativos [Internet]. Universitat de València; 2016 [citado 10 de febrero de 2020]. Disponible en https://dialnet.unirioja. es/servlet/tesis? codigo $=76429$.

Zabalegui, A., Rodríguez, E., Ramírez, A.M., Pulpón, A., López, L., Izquierdo, M.D., Gual, P., González-Valentín, A., Gallart, A., Díaz, M., Corrales, E., Cabrera, E. y Bover, A. Revisión de evidencias: cuidadores informales de personas mayores de 65 años. Evidentia 2007 ene-feb; 4(13). Disponible en http://www.index-f.com/evidentia/n13/291articulo. php [ISSN: 1697-638X]. 